Proyecciones

Vol. 21, Nº 2, pp. 127-139, August 2002.

Universidad Católica del Norte

Antofagasta - Chile

\title{
PROCEDIMIENTO MATEMATICO \\ PARA EVALUAR EL TRANSITO HACIA EMPRESAS DE CLASES. GENERALIDADES
}

\author{
MARIA DE LOURDES ARTOLA PIMENTEL \\ Universidad de Matanzas - Cuba
}

\begin{abstract}
En el presente se destacan como aspectos novedosos la utilización creativa de los conceptos y principios de las relaciones de sobreclasificación borrosa para la determinación de los indices de credibilidad experto estadío, la propuesta de la autora de enfocar el problema de integración de las preferencias de los expertos como un problema multicriterios borroso, el análisis de éstas como conjuntos borrosos y la definición de indicadores como proximidad, alejamiento, debilidades, fortalezas, entropía o desorden, así como la propia manera de clasificar.
\end{abstract}

Palabras claves : Multicriterio borroso, sobreclasificación. 


\section{Introduction}

La evaluación constituye una etapa imprescindible en la concepción de acciones de mejoramiento en cualquier sistema, es un inputs para su desarrollo y desenvolvimieto. La empresa definida como tal requiere de procedimientos para autoevaluarse. El sistema empresa transita por varios estados en dependencia de su grado de desarrollo.

Desde el punto de vista matemático la evaluación referida se caracteriza por tener múltiples criterios generalmente en conflicto por el grado desigual de desarrollo en que éstos suelen encontrarse, suscitándose un problema multicriterio, de otra parte en el nuevo contexto decisional de los fenómenos empresariales, se pasa de la aleatoriedad a la borrosidad, debido a que por su naturaleza e intuición la imprecisión se formaliza a través de situaciones en las que existe graduación entre pertenencia absoluta y la no pertenencia.

La evaluación se sustentará en criterios de los expertos evaluadores, utilizando como método principal de captación la encuesta, a través de la cual cada experto valorará el cumplimiento o no del criterio-estadío a través de un formato como el que sigue:

No estoy de acuerdo $\quad$ No estoy de acuerdo ni en desacuerdo

$\begin{array}{llllllll}1 & 3 & 4 & 5 & 6 & 7 & 8 & 9\end{array}$

Estoy de acuerdo

(A)

Figura 1. Continuum que facilitará la evaluación del experto. Elaboración propia

\section{Fase de Procesamiento}

Tiene como entradas las evaluaciones hechas por los expertos, en esta se estima el empleo de métodos y técnicas de la Teoría de la Decisión a fines al problema de evaluar basado en preferencias, referido a lo cual se precisa que la autora incorpora el principio de construcción de la relación de sobreclasificación de los Métodos Electre que se basa en la heurística ampliamente aceptada de la regla de mayoría y el respeto a las minorías significativas que aquí se representa por el principio de concordancia-discordancia, el que se aplica al determinar los índices de credibilidad por expertos individuales y grupal. 
Consta de tres módulos: cálculo de la matriz de pertenencia del experto, creación de la matriz global pertenencia, análisis y clasificación que se describirán a continuación:

\section{CÁLCULO DE LA MATRIZ DE PERTENENCIA DEL EXPERTO}

Dadas:

- Un conjunto C de empresa-criterio-estadío $\left(c r_{j}\right)_{j=1 . . p+3}$, predefinidos como

$\mathrm{C}=\{$ criterio procesos claves (1..p) criterio resultado clientes, criterio resultado empleados, criterio resultado globales\} en los estadíos confiable, competente y de clase.

- Un conjunto de subcriterios-estadíos $\{g j\}_{j=1,2, \ldots, m}$ que se seleccionan en la fase de definición, $c r \in C$, y para lo cuales se determinará el grado de credibilidad de su cumplimiento.

- Un conjunto $E=\{e i\}$ de $n$ expertos seleccionados en la fase de definición y las evaluaciones $\left\{g_{j}(e i)\right\}, \forall e_{i} \in E$ y $\forall g_{j}$

- Un umbral de indeferencia $q$, referidos al cumplimiento o no del subcriterio.

- Un umbral de preferencia p, referido al cumplimiento o no del subcriterio

- Un umbral de veto v.

Para cada empresa-criterio-estadío-experto se hará el siguiente procesamiento.

Teniendo en cuenta el continuum no estoy de acuerdo-estoy de acuerdo se define: N como el artículo -No estoy de acuerdo ni en desacuerdo con el cumplimiento del criterio-estadío-; A como el artículo -Estoy de acuerdo- y D en el otro caso.

Se define un índice de concordancia c y un índice de discordancia $d_{j}$ de la siguiente manera:

$$
c=\frac{1}{m} \sum_{j=1}^{m} c_{j}\left(e_{i}\right)
$$


donde

$$
\begin{aligned}
& c_{j}\left(e_{i}\right)=\left\{\begin{array}{llll}
1 & \text { si } & g_{j}\left(e_{i}\right) & \text { es } \mathrm{A} \\
\frac{g_{j}\left(e_{i}\right)-q}{p-q} & \text { si } & g_{j}\left(e_{i}\right) & \text { es } \mathrm{N} \wedge g_{j}\left(e_{i}\right) q \\
0 & & \text { en el otro caso } &
\end{array}\right.
\end{aligned}
$$

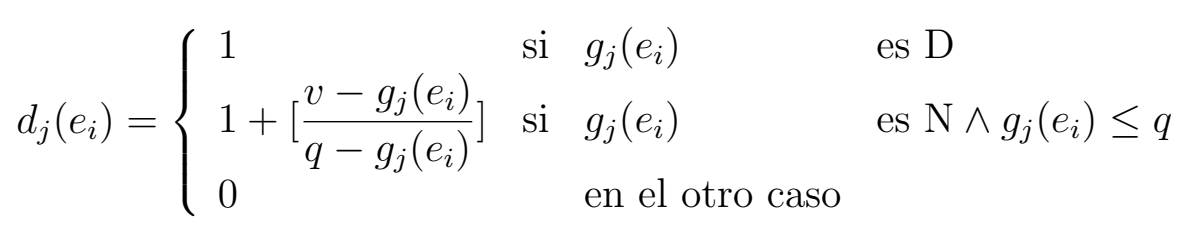

El índice de concordancia c se considera como una medida de los argumentos a favor de la aseveración el criterio-estadío-empresa está cumplido, mientras que el índice de discordancia $d_{j}$ mide la "robustez" de las razones para refutar esta aseveración.

Conocidos los componentes, se define el índice de credibilidad del continuum no estoy de acuerdo o estoy de acuerdo $S\left(e_{i}, c r_{j}\right)$ para el empresa-criterio-estadío-experto de la siguiente manera:

$$
S\left(e_{i}, c r_{j}\right)= \begin{cases}c & \text { si } \quad d_{j} \leq c \\ c * \prod_{j \in J} \frac{1-d_{j}}{1-c} & \text { en el otro caso }\end{cases}
$$

donde $\mathrm{J}$ es el conjunto de criterios $\mathrm{j}$ tal que $d_{j}>c$. Se puede interpretar a $\mathrm{S}$ como una medida de la credibilidad del experto decisor acerca de la aseveración o pertenencia a un determinado estadío del criterio que se evalúa. Como resultado de este paso se obtendrá una matriz criterio-estadío para cada experto.

Cuando los criterio-estadíos se diferencia en su grado de importancia entonces debe modificarse el índice de concordancia, en cuyo caso se definiría como sigue:

$$
c=\frac{1}{W} \sum_{j=1}^{m} c_{j}
$$

donde

$$
W=\sum_{j=1}^{m} w_{j}
$$


siendo $w_{j}$ el "peso"

\section{CREACIÓN DE LA MATRIZ GLOBAL PERTENEN- CIA}

La mayoría de los métodos de decisión multicriterio fueron creados en un contexto monousuario. Sin embargo, el pretender utilizar la estructura de estos métodos en un contexto de decisión en grupo, a la que el objeto de estudio condiciona, no parece que sea en sí misma una buena decisión. De inicio, estos métodos consideran una matriz de evaluación por cada experto, para después tratar de integrar todas estas matrices en una sola, lo cual se puede hacer por dos caminos: mediante operaciones aritméticas (evaluación promedio, evaluación max, evaluación min,...) o pidiendo al grupo de expertos que llegue a una matriz consensuada.

Obviamente el primer camino no es recomendable porque no se pueden justificar operaciones aritméricas de estos tipos, además de que la matriz resultante no corresponde a nada real; el segundo camino es mejor, dado que producirá una matriz que corresponde con la realidad del problema; no obstante, encontrar un punto de consenso de un grupo puede resultar un proceso largo y difícil, en caso de que sea posible.La idea que se utilizará por la autora consiste en que cada experto exprese sus propias preferencias y creencias, se integren de manera individual, y después se integren las de todos los expertos en uno global. Este enfoque tiene la ventaja de tomar en cuenta las preferencias de todos los actores [Macharis, 1998]. Dado que un esquema compensatorio no es el más apropiado para la decisión en grupo se considera por la autora que en estos casos debe utilizarse la heurística más natural basada en reglas de mayoría combinada con concesiones a las minorías significativas, enfatizado por el objeto de la evaluación.

La información de entrada será una familia de matrices de evaluación, una para cada criterio empresa conteniendo del índice de credibilidad estadío-experto, obteniéndose una matriz global por criterio empresa como resultado.

Dados:

- Conjunto de expertos decisores $E=\left\{e_{j}\right\}_{j=1 \ldots n}$ 
- Conjunto $\mathrm{C}$ de empresa-criterio-estadío $\left(c r_{j}\right)$, predefinidos como $\mathrm{C}=\{$ criterio proceso clave $1, \ldots$, proceso clave $\mathrm{p}$, criterio resultado clientes, criterio resultado empleados, criterio resultado globales

- Matriz de entrada para cada empresa-criterio-estadio-expertos.

- Un valor $I E_{j}$ que expresa el grado de experticidad del experto j evaluado en la fase de definiciones.

- Los umbrales de preferencia $\alpha$ y de indiferencia $\beta$ nivel de veto $N_{v}$ consensuados para el conjunto $E=\left\{e_{j}\right\}$ y que constituyen distinciones de las preferencias grupales

- Un conjunto de estados $F=\{$ confiable, : competente, de : clase $\}$

Se definen :

- Coalición de Concordancia

$$
C C=\left\{e_{j} \in E / S\left(e_{i}, c r_{j}, f_{k}\right)>\alpha\right\}
$$

- Coalición de Veto

$$
V=\left\{e_{j} \in E / S\left(e_{i}, c r_{j}, f_{k}\right)<\beta\right\}
$$

- Coalición de Incomparabilidad

$$
R=\left\{e_{j} \in E /: e_{j} \quad \text { no }:: \text { voto }\right\}
$$

Sean:

$$
c\left(c r_{i}, f_{i}\right)=\frac{1}{W} \sum_{j \in C C} I E_{j}
$$

donde

$$
W=\sum_{j \in C C \bigcup V} I E_{j}
$$




$$
v t_{j}=\left\{\begin{array}{ccc}
1 & \text { si } I E_{j} \leq \overline{I E} \\
1.5 & \text { si } I E_{j}>\overline{I E}
\end{array}\right.
$$

siendo $\overline{I E}$ el peso o importancia promedio de los expertos.

$$
\begin{aligned}
& N v\left(c r_{i}, f_{k}\right)=\left\{\begin{array}{cc}
\frac{\sum_{j \in C C \bigcup V} w_{j}-\sum_{j \in R} w_{j}}{\sum_{j \in C C \bigcup V} w_{j}} & \text { si } \sum_{j \in C C \bigcup V} w_{j} \geq \sum_{j \in R} w_{j} \\
0 & \text { si } \sum_{j \in C C \bigcup V} w_{j}<\sum_{j \in R} w_{j}
\end{array}\right. \\
& D_{g}\left(c r_{i}, f_{k}\right)=\left\{\begin{array}{cl}
\frac{\sum_{j \in V} v t_{j}}{N v} \text { si } & \sum_{j \in V} v t_{j}<N v \\
1 & \text { de otramanera }
\end{array}\right.
\end{aligned}
$$

Entonces el índice de credibilidad global $S g\left(c r_{j}, f_{k}\right)$ se define de la siguiente manera:

$$
S g\left(c r_{j}, f_{k}\right)=c\left(c r_{j}, f_{k}\right) *\left[1-D g\left(c r_{j}, f_{k}\right)\right] * N v\left(c r_{j}, f_{k}\right)
$$

y se interpreta $S g\left(c r_{j}, f_{k}\right)$ como una medida de la credibilidad grupal de la pertenencia del criterio $c r_{j}$ al estadío $f_{k}$.

Sea entonces $M G(C, F)$ la matriz formada por los vectores $\overline{S g}(C, F)$ que se le ha denominado matriz global de pertenencia. 


\section{ANÁLISIS Y CLASIFICACIÓN}

1. Cálculo de los vectores distancia Hamming

Dada la matriz $M G(C, F)$ y considerando a sus vectores filas conjuntos borrosos:

$$
\left\lfloor\mu_{1} \ldots \mu_{p}\left|\mu_{p}+1\right| \mu_{p}+2\left|\mu_{p}+3\right|\right.
$$

Donde $\mu_{i} \in[0,1]_{i=1,2, \ldots . . m}$, y se le ha llamado índice de credibilidad tal como es usual en la matemática de la incertidumbre, considerando que el valor $\mu_{i}=1$ es el ideal que se identifica con pertenencia estricta a un estadío para el criterio dado entonces un vector fila donde $\mu_{i}=1 \forall i$ sería un vector de pertenencia ideal para el estadío. La Distancia Hamming [Gil Aluja, 1998],[Garza Ríos, 2000], permite medir el nivel de entropía de cada vector fila respecto a ese ideal y se calcula como sigue:

$\delta_{i}=\frac{1}{m} \sum_{j=1}^{p+3}\left|\mu_{D}-\mu_{j}\right| \forall i \in[1 . .3]$

donde:

$p+3$ : número de criterios a considerar.

$\mu_{D}$ : valuación del criterio en el perfil ideal.

$\mu_{j}$ : valuación del criterio j para la alternativa que se analiza.

Mientras esa distancia sea mínima habrá más razones para aceptar que la empresa es de esta clase. De manera análoga se trabaja por columnas de la matriz denotando procesos y resultados que constituyen fortalezas o debilidades para la organización. El análisis puede hacerse de forma independiente entre procesos y resultados.

Si

$\exists i_{\text {maximo }} M G(i, j)=1 \quad \forall i<i_{\text {maximo }}$

entonces las empresa se clasifica según el $i_{\text {maximo }}$ estadío, en este caso $\delta_{i}=0$, de lo contrario no se cumplen los requisitos para situarla en ninguno de los tres estadíos y se determina la proximidad para cada criterio de la siguiente manera: 
Considerando la matriz $M G(i, j)$ como una matriz borrosa compuesta por conjuntos borrosos identificados como sus vectores filas o vectores columnas entonces:

2. Se determina la proximidad como el conjunto borroso intersección de a las filas de la matriz a partir del referencial ideal D definido anteriormente, me determina el estadío más cercano, el que se corresponde con el subíndice i de los elementos que conforman el subconjunto intersección definido. La coincidencia en el índice i para diferentes criterios-empresa muestra claras evidencias acerca de la cercanía a un determinado estadío.

3. Se determina el alejamiento como el conjunto borroso unión de las filas de la matriz, tomando como referencial el ideal D . El alejamiento-estadío se define para criterio y me determina el estadío más lejano, el que se corresponde con el subíndice i de los que conforman el subconjunto unión. La concordancia en los valores del índice i muestra claras evidencias de que ese es el estadío más distante.

4. Se determinan las fortalezas como un conjunto borroso intersección de las columnas de la matriz, tomando como referencial el ideal definido anteriormente, la fortaleza determina el o los criterios más cercanos al ideal que se corresponde con el subíndice j de los que conforman el subconjunto intersección definido. Si los índices coinciden quiere decir que la fortaleza es concordante y marcada hacia un criterio-empresa.

5. Se determinan las debilidades como un conjunto borroso unión de las columnas de la matriz, tomando como referencial el ideal definido anteriormente. Las debilidades me determinan los criterios más alejados del ideal que se corresponde con el subíndice i de los que conforman el subconjunto intersección definido. Si los índices coinciden quiere decir que la debilidad es concordante y marcada.

6. Se determina la entropía relativa para un $\alpha$ prefijado

Dado $\alpha$ determinar la matriz borrosa 


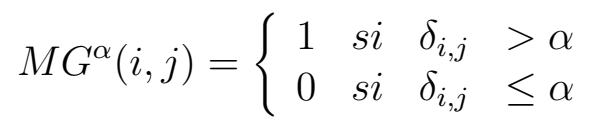

Conclusiones:

Se da una propuesta de solución al problema de origen y se demuestra la factibilidad de resolver el mismo a través del uso de las herramientas brindadas por la Teoría de la Decisión con enfoque descriptivo y considerando además el enfoque borroso

\section{References}

[1] Arlington Software Corporation: AHP In Perspective, Reporte técnico ARL98 - ER-D01. WWW.arlingsoft.com, (1998).

[2] Brans J. P., Mareschal, B., y Vincke Ph.:PROMETHEE: a new family of outranking methods in MCDM, Operational Research, North Holland, pp. $477-490,(1984)$.

[3] Buchanan, J.T. y Henig M.J.:Objectivity and Subjectivity in the Decisión Making Process, Reporte técnico, Department of Management Systems, University of Waikato, www.mngt.waikato.ac.nz/depts/mnss/john/subobj1.htm, (1997).

[4] Cuétara, Leonardo; Modelo de Evaluación de Empresas Transporte Turístico. Tesis para optar por el grado de Doctor en Ciencias Económicas. Ciudad de La Habana, Cuba, (2000).

[5] D’Avignon y Vincke, G., y Martel J. M.: Selecting Experts for Projects Evaluations, Operational Research'84, Elseiver Science Publisher, (1988).

[6] Diaz L.C., Costa J. P., y Clímaco J N.: A Parallel Implementation of the PROMETHEE Meted, European Journal of Operational Research, Vol. 104. pp. 521 - 531, (1998). 
[7] Fernández E.: El Método EDIPO para la Ayuda a la Decisión Multicriterio, UPIICSA Tecnología Ciencia y Cultura, Vol III, No.19, pp.25 - 30, (1999).

[8] Fodor y Roubens M.: Fuzzy Preference Modeling and Multicriteria Decision Support, Kluwer, Dordrecht, (1994).

[9] French S.:Decision theory: An introduction to the mathematics of rationality, Halsted Press, New York-Chichester-BrisbaneToronto, (1986).

[10] Gil Aluja, J.: Nuevas técnicas para la gestión de empresas. Retos Empresariales para (1998), Management Empresa, Universidad de Barcelona, (1997).

[11] González Santoyo, F.; Flores Romero; J.J., Flores; Flores Romero, B.: La incertidumbre en la evaluación financiera de las empresas; Morevallado Editores, Morelia, México, (2000).

[12] Hair J.E.; Anderson, R.E.; otros: Análisis Multivariante, Prentice Hall, Iberia Madrid, (1999).

[13] Kaufman A.; GilAluja;Terceño A.: Matemática para la economía y la gestión de empresas, Ediciones Foro Científico, S.L. Vol. 1, Barceona, España, (1994).

[14] Keeney y Raiffa, .: Decisions with multiple objectives. Preferences and Value Tradeoffs. New York : Jonh and Sons, (1976).

[15] Leyva J.C: Aplicación de los algoritmos genéticos a la solución del problema de decisión multicriterio individual y en grupo Tesis en opción del grado de Doctor en Ciencias de la Computación, Universidad de Sinaloa, México, (2001).

[16] Marrero, F.: Toma de decisiones multicriterio en la cadena de corte, alza y tiro de caña de azúcar, Evento Logística, Cuba, (2000).

[17] Marrero, F.: Procedimiento para la toma de decisiones logísticas con enfoque multicriterio en la cadena de corte, alza y transporte 
de la caña de azúcar. Aplicaciones en CAI de la provincia de Villa Clara, Tesis en opción del grado científico de Doctor en Ciencias Técnicas, La Habana, (2001).

[18] Pardalos, Siskos C. y Zopounidis, C.,: Advances in Multicriteria Análisis, Kluwer Academic Publishers, (1995).

[19] Pomerol J. Ch y Barba-Romero: Artificial Inteligence and $\mathrm{Hu}-$ man Decision Making, 14th European Conference on Operational Reseach, (1993).

[20] Robins, E.S. :An Investigation into the Efficacy of the Consistency Ratio with Matrix Order-Limits of the AHP, Reporte técnico ARL97-ER-D01. Arlington Software Corporation. www.arlingsoft.com, (1997).

[21] Roy B.y Bertier: La Methode ELECTRE II". M Ross (ed) OR'72. North - Holland Publishing Company, (1973).

[22] Roy B.: The outranking approach and the foundations of ELECTRE methods. Bana e Costa, C.A., (ed.) Reading in multiple criteria decision aid. Springer-Verlag, Berlin. pp. 155 - 183, (1990).

[23] Roy B.:Multicriteria Methodology for Decision Aiding, Kluwer , (1996).

[24] Saaty, .T.L.: The Analytic Hierarchy Process. New York : McGraw Hill (1980).

[25] Salminen P., Hokkanen J., y Lahdelma R.: Comparing multicriteria methods in the context of environmental problems, European Journal of Operational Research Vol. 104, pp. 485 - 496, (1998).

[26] Stewart, B.: A Science of Social Issues, (1992).

[27] Trejos, :Método de Relaciones Binarias de Sobreclasificación que usa una Familia de Funciones de Utilidad, Disertación Doctoral, Universidad Nacional Autónoma de México, (1991). 
[28] Vincke: Outranking Approach, Reporte técnico IS-MG 98/08, Institut de Statistique et de Recherche Opérationnelle, Universite Libre de Bruxelles. Serie: Mathématiques de la Gestion, (1998).

Received : Mayo 2002.

Msc. Lic. María de Lourdes Artola Pimentel

Facultad de Ingeniería Informática

Universidad de Matanzas

C. P. 44740

Cuba

e-mail : mlap63@yahoo.es 